Supporting Information

\title{
Proton Transport in Mesoporous Silica SBA-16 Thin Films with Three-Dimensional Cubic Structures
}

\author{
Junho Hwang ${ }^{\dagger}$ and Hirofumi Daiguji ${ }^{\dagger}$
}

$\dagger$ Division of Environmental Studies, Graduate School of Frontier Sciences, The University of Tokyo

5-1-5 Kashiwanoha, Kashiwa 277-8563, Japan

*To whom correspondence should be addressed.

E-mail: daiguji@k.u-tokyo.ac.jp, Phone: +81 47136 4658, Fax: +81 471364659. 


\section{Restoration of electric potential barriers by deprotonation reaction}

To elucidate the effect of surface charge density of mesopores on $I-V$ curves, the $I-V$ curves were measured by changing the soaking duration of a nanofluidic device in deionized (DI) water (18.2 $\mathrm{M} \Omega \cdot \mathrm{cm})$. Figure $\mathrm{S} 1$ shows the measured $I-V$ curves for four different soaking durations. Following the measurement of the $I-V$ curve of $1 \mathrm{M} \mathrm{HCl}$ aqueous solution, a nanofluidic device was immersed in DI water for several days to restore silanol groups in the pore surface. The $I-V$ curve of $10^{-7} \mathrm{M}$ $\mathrm{HCl}$ aqueous solution was measured every $24 \mathrm{~h}$. Despite the extremely low concentration, the measured $I-V$ curve was almost linear at 1 day, since the silanol groups were not yet fully restored from the previous protonation. Eventually, however, the measured $I-V$ curve became nonlinear because the silanol groups were restored by deprotonation, and the electric potential barriers increased. After 4 days, the silanol groups were fully restored and the $I-V$ curve agreed with the measured one. These results suggest that the $I-V$ curve of $\mathrm{HCl}$ aqueous solutions was sensitive to the surface state of mesopores, and control of the surface state was crucial to elucidate the characteristics of $I-V$ curves.

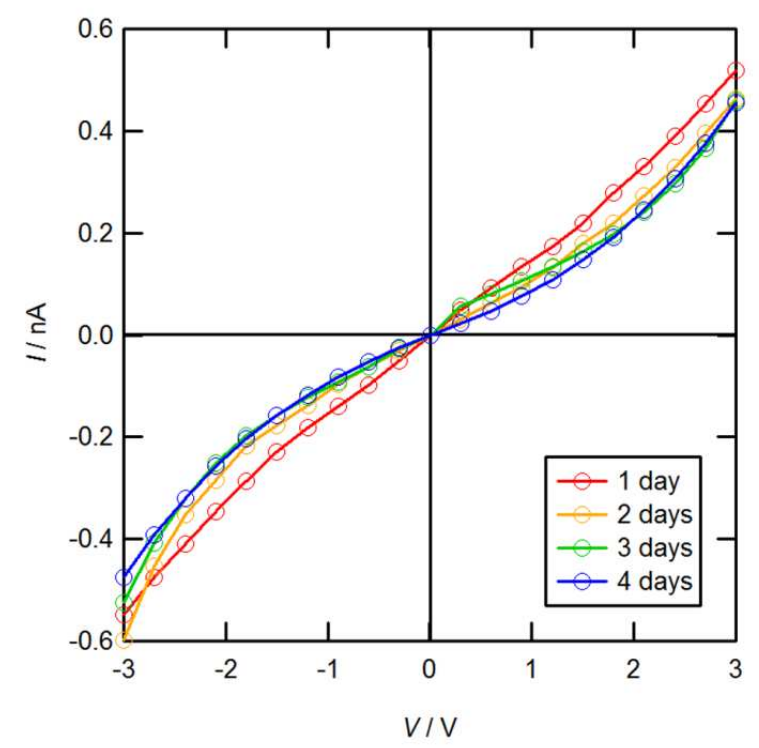

Figure S1. Effect of soaking duration of a nanofluidic device in DI water on current vs. electric potential bias $(I-V)$ curves. 